\title{
Sistema de control de pago de matrículas y aranceles de la Universidad de las Regiones Autónomas de la Costa Caribe Nicaragüense, Recinto Las Minas, 2015
}

Esmir Noel Roque Castellano ${ }^{1}$ Freddy Betzael Vargas Moreno ${ }^{2}$ Elkis Aismara Ortega Carrasco ${ }^{3}$

\section{Resumen}

Se desarrolló un sistema de control de pagos de matrículas y aranceles de los estudiantes en la URACCAN, Recinto las Minas Siuna, que almacena la información de forma efectiva. Automatiza, registra y brinda información en tiempo real. Es un estudio aplicativo con enfoque cuantitativo. La fuente primaria fue el personal administrativo dedica al manejo de control de pagos de matrículas y aranceles, como fuente secundaria todos los registros, formatos y documentos que utiliza el área de administración. Se creó un Sistema de Base de Datos con el gestor, Structured Query Language (SQL) Server 2014, está conformado de diez tablas, debidamente relacionadas y normalizadas que evita la redundancia de datos. La interfaz gráfica se diseñó en Visual Studio 2010, con el lenguaje de programación Visual Basic y se crearon 22 formularios, que permite al usuario interactuar con la base de datos de forma sencilla y segura. El sistema de información se implementó en el área de Administración que pasó por un periodo de prueba, de tal manera que se identificaron los posibles errores y se corrigieron a través de la reingeniería del sistema.

Palabras clave: Ingresos, registro; sistema de control.

\section{Resumen}

A system of control of payments of tuition and fees of the students was developed in URACCAN, Siuna Campus, that stores the information in an effective way. It automates, registers and provides information in real time. This study is based on a quantitative approach.

The primary source was the administrative staff dedicated to the management and control of payments of tuition and fees; and as a secondary source we had all the registrations, formats and documents used by the administration area. A Database System was created with the manager, Structured Query Language (SQL) Server 2014, which is made up of ten tables, properly related and standardized, that avoids data redundancy.

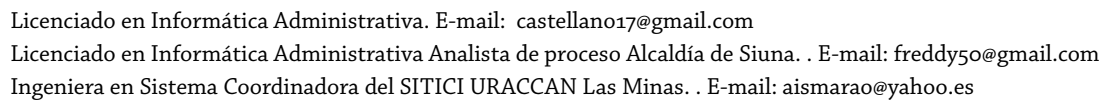


The graphical interface was designed in Visual Studio 2010, with the programming language Visual Basic and 22 forms were created, which allows the user to interact with the database in a simple and safe way. The information system was implemented in the Administration area that went through a trial period, in such a way that the possible errors were identified and corrected through system reengineering.

Keywords: Income, registration; control system.

\section{Introducción}

A nivel mundial los sistemas de información con base de datos de altas, bajas y consultas tienen un gran auge, porque estos sistemas tienen el mejor rendimiento y agiliza el trabajo en las empresas, además la calidad es única. En la Región Autónoma de la Costa Caribe Norte, se poseen sistemas de información en la mayoría de las instituciones estatales y también en algunos organismos no gubernamentales, pero a pesar de ello, existe una gran demanda de estos sistemas sobre todo en el sector comercial.

En el área de administración de la URACCAN, Recinto las Minas, Siuna, se vio la necesidad de instalar un sistema para el control y manejo de los pagos de aranceles y matrículas de los estudiantes, esto hace que dicho proceso se realice de forma lenta, lo que provoca la redundancia e inconsistencia de datos, debido a que no se tiene una información eficaz para la toma de decisiones.

Con el estudio, se realizó un Sistema de Información con Bases de Datos el cual permite obtener un control de pagos de matrículas y aranceles de los estudiantes, el que facilita obtener y mantener de forma actualizada, rápida y sin redundancia el control de dichos ingresos de matrículas y aranceles, en tiempo real y segura.

\section{Revisión de literatura}

\section{Generalidades}

Según Peña (2006, p.7), un Sistema de Información de Base de Datos es un conjunto de elementos interrelacionados con el propósito de prestar atención a las demandas de información de una organización, para elevar el nivel de conocimientos que permitan un mejor apoyo a la toma de decisiones y desarrollo de acciones.

Por información se entienden los datos que se han moldeado en una forma significativa y útil para los seres humanos. En contraste, los datos son secuencias de hechos en bruto que representan eventos que ocurren en la organización, eso en el entorno físico antes de ser organizados y ordenados en una forma que las personas puedan entender y utilizar de manera efectiva (Laudon \& Laudon, 2008, p.14). 
Según Castaño y Plattini (1999), los sistemas de gestión de base de datos, se ocupan del tratamiento, definición, actualización y recuperación de datos estructurados (p.15). Mientras los sistemas de recuperación de información se ocupan del tratamiento de datos no estructurados (Castaño \& Plattini, 1999, p.14).

\section{Sistemas de base de datos}

Según Osorio (2008, p.13), un sistema de base de datos es un conjunto de elementos interrelacionados y una serie de programas que permiten a varios usuarios tener acceso a estos archivos. Un sistema de base de datos proporciona un lenguaje de definición de datos para especificar el esquema y un lenguaje de manipulación de datos para expresar las consultas y modificaciones (Silberschartz, 2002, p.7).

\section{Creación de la Base de Datos}

El término de base de datos se refiere a una organización de componentes que definen y regulan la recolección, almacenamiento, administración y uso de los datos dentro de un ambiente de base de datos. Desde el punto de vista de administración general, este sistema se compone de las cinco partes principales: hardware, software, personas, procedimientos y datos (Kendall \& Kendall, 2005, p.14).

\section{El lenguaje: Structured Query Language (SQL)}

Idealmente, un lenguaje de base de datos debe permitir crear bases de datos y estructuras de tabla para realizar tareas de administración de datos básicas (agregar, eliminar y modificar) y realizar consultas complejas diseñadas para transformar los datos sin procesar en información útil. Además, debe realizar funciones básicas con un mínimo esfuerzo del usuario, para manejar su estructura y sintaxis de comandos. Por último, debe ser portátil, es decir, debe ajustarse a un estándar básico (Castaño \& Plattini, 1999, p.129).

Es importante destacar que éste es el gestor de bases de datos más actual, razón por la cual lo hemos elegido para nuestro objeto de estudio, además es el que más se apega a las necesidades de almacenamiento de nuestro estudio, esto lo confirma Osorio (2008, p.13), al mencionar que Structured Query Language (SQL) Server, es la plataforma para bases de datos que más se utiliza en el procesamiento de datos a gran escala en todos los ámbitos de aplicación.

\section{Interfaz}

Una interfaz es un dispositivo que permite comunicar dos sistemas que no hablan el mismo lenguaje. Restringido a aspectos técnicos, se emplea el término interfaz para 
definir el juego de conexiones y dispositivos que hacen posible la comunicación entre dos sistemas (Lamarca, 2014).

Las interfaces gráficas de usuario (GUI) permiten la manipulación directa de la representación gráfica en pantalla, la cual se puede realizar con la entrada del teclado, una palanca de juego o el ratón (Kendall \& Kendall, 2005 p. 503).

\section{Implementación}

La implementación de sistemas implica crear o adquirir los diversos componentes del sistema como: hardware, software y bases de datos, definidos en el paso de diseño, su montaje y la puesta en operación del nuevo sistema. El propósito del mantenimiento y revisión de sistemas es inspeccionar y modificar el sistema a fin de que responda a los cambiantes necesidades de la empresa (Ralph \& George, 2013, p.29).

En general, existen diversos enfoques para la incorporación del ambiente de implementación a la arquitectura del sistema: como agregar clases abstractas o interfaces que luego se especializarán según el ambiente de implementación particular; la instalación objetos especializados que administren los aspectos particulares del ambiente de implementación; el poder configurar múltiples versiones del sistema correspondientes a diferentes plataformas (Weitzenfeld, 2005, p.499).

Se realizó en el área de Administración en URACCAN Recinto Las Minas Siuna, ubicada en el barrio "Pedro Joaquín Chamorro" sector 3 del municipio de Siuna, Nicaragua. Es un estudio aplicativo con un enfoque cuantitativo, porque se desarrolló un sistema de información para resolver el problema de ingresos y egreso de los pagos que realiza la comunidad estudiantil en el área de Administración. Se tomaron en cuenta los pagos de matrículas y aranceles de los estudiantes y se excluyeron, todos los pagos que no se corresponden a lo que prioriza el estudio. Las fuentes primarias fueron, el personal administrativo que se dedica al manejo del control de pagos de matrículas y aranceles; las secundarias: registros, formatos y documentos que utiliza el área de administración: libros para registrar el pago de matrículas y aranceles. Las técnicas que se utilizaron fueron: las entrevistas semi-estructuradas y revisión documental.

\section{Procesamiento de la información}

Una vez realizada la revisión documental y entrevistas se definen las tablas, campos, tipos de datos, tamaños de los campos de la base de datos y la estructura de los formularios que conformaron el sistema. Posteriormente, se procedió a la creación de la base de datos, la interfaz de usuario y la implementación del sistema. Para la creación de la base de datos, primero se realiza la instalación del Sistema Gestor de Bases de Datos, Structured Query Language SQL Server 2014 por ser robusto, estable y actualizado. 
Se utiliza el modelo de datos Relacional, para lo cual se realiza un esquema, con el Gráfico Relacional. Consecutivamente, se procede a crear la base de Datos, en el Sistema Gestor de Base de Datos, Structured Query Language SQL Server 2014.

La interfaz de usuario se hizo en el lenguaje de programación Visual Basic, integrado en el paquete Visual Studio, versión 2013. Se implementó el uso de usuarios y contraseñas para la seguridad del sistema. Desde los formularios, se puede crear respaldo de la base de datos que forma parte de la seguridad del sistema.

Una vez que finaliza el desarrollo del sistema, se verifican los requerimientos de hardware donde está instalado el sistema, el cual incorpora la clave de acceso y la encriptación de la información. Se procedió a la verificación del usuario final para identificar los posibles problemas o cambios necesarios de acuerdo a las necesidades para el desarrollo y desempeño; además, se realizaron pruebas de entradas y salidas ya sea en registro nuevos y los reportes; estas pruebas se hicieron con datos reales para comprobar los funcionamientos y verificar posibles errores que se corrigieron mediante reingeniería.

La reingeniería consiste, que a partir de los errores, se identifican las causas, se corrige el error y se verifica nuevamente su funcionamiento. Cada observación por el usuario final, se toma en cuenta para mejorar la interacción entre este y el sistema.

\section{Resultados y discusión}

El sistema lo conforman una base de datos en Structured Query Language (SQL) Server 2014, contiene diez tablas en total, por seguridad está protegida por un usuario y contraseña. Desde la interfaz gráfica, se puede realizar copia de seguridad de la base datos, la que servirá para restaurarlo, en caso de una falla del sistema.

Visual Basic integrado en Visual Studio 2013, fue el lenguaje de programación para el desarrollo de la interfaz gráfica, con veintidós formularios los que interactúan con la base de datos y realiza funciones como agregar, actualizar, eliminar registros, reportes del estado de cuenta sobre pagos de aranceles y matrículas, y la migración de las consultas y tablas a Excel, a través de un diseño visual amigable y adecuado, para facilitar la utilización del sistema.

El sistema se implementa en el área de Administración de la URACCAN Las Minas Siuna. El que se verifica y se somete a prueba para identificar algunos problemas y fallas que fueron corregidos mediante reingeniería.

\section{Creación de la Base de Datos}


Se instala el Sistema Gestor de Base de Datos, Structured Query Language (SQL) Server 2014, cumple con los requisitos que establece Microsoft (2014), con al menos 6 Gigabyte en disco duro, 512 MegaByte (MB) de Random Access Memory (RAM) y un procesador de 1,4 GigaHertz (GHz).

EL Structured Query Language (SQL) Server 2014, tiene muchas ventajas, una de ellas es que ayudará a suplir las necesidades del área para el que fue creada, de manera rápida, con mayor facilidad y se puede almacenar gran cantidad de datos.

Los planteamientos anteriores están de acuerdo con lo estipulado por Osorio (2008, p.77), al mencionar que Structured Query Language SQL Server, es la plataforma para bases de datos que más se utiliza en el procesamiento de datos a gran escala en todos los ámbitos de aplicación.

\section{Interfaz de usuario}

La interfaz gráfica de usuario fue diseñada en el paradigma de la Programación Orientada a Eventos. Como resultados, se obtuvieron, veinte y dos (22) formularios que conforman la totalidad de la interfaz, que permite agregar, eliminar y actualizar la información de las bases de datos, así como la generación de consultas y reportes.

El desarrollo de sistema con el uso de Visual Studio 2013, permite desarrollar un sistema informático para el control de pagos de matrículas y aranceles. Esto coincide con Visual Studio, (2014), que confirman que es una colección completa de herramientas y servicios que admite crear una gran variedad de aplicaciones, tanto para plataformas de Microsoft, como para otras. Visual Studio conecta también, todos los proyectos, equipos y partes interesadas.

\section{Implementación del sistema}

Después de la instalación del sistema, se puso a prueba con el usuario final con fines prácticos, que consiste en cómo acceder al sistema, agregar un nuevo estudiante, consultas y reportes. El período de prueba presentó algunos problemas asociados con la instalación, estas fallas fueron corregidas, a la vez que se verifica, si el sistema funcionará tal y como se esperaba.

Esto concuerda, con lo que afirman Ralph y George (2013, p.29), quienes establecen que el propósito del mantenimiento y revisión de sistemas es inspeccionar y modificar el sistema a fin de que responda a las cambiantes necesidades de la empresa. 


\section{Conclusiones}

El estudio se realiza en el área de Administración de URACCAN Recinto Las Minas, Siuna, tuvo como resultado el desarrollo de un sistema de control para el pago de matrículas y aranceles el cual ayuda a agilizar la información y el rendimiento de dicha misma.

Se creó un Sistema de Base de Datos en el Gestor, Structured Query Language (SQL) Server 2014, está conformada de diez tablas debidamente relacionadas y normalizadas lo que evita, la redundancia de datos. La interfaz gráfica se diseñó en Visual Studio 2013 con el lenguaje de programación Visual Basic en el que se crea 22 formularios, que le permite al usuario interactuar con la base de datos de forma sencilla y segura.

Los formularios que se crearon, por su sencillez y diseño son agradables para la vista como para el uso de los mismos. Esto permite realizar las altas, bajas y cambios de información, así como las consultas impresas y digitales.

El sistema de información que se implementa en el área de Administración pasa por un período de prueba y así verifica posibles errores, los que se corrigen a través de la reingeniería del sistema de manera que su función sea exitosa.

\section{Recomendaciones}

\section{A los usuarios del sistema}

- Proceder con la introducción de los datos de cada estudiante activo. Mantener de forma adecuada y regular el control de pagos de matrículas y aranceles.

\section{AI Recinto URACCAN las Minas, Siuna}

- Incluir en el recibo el número de carnet de cada estudiante.

- Utilizar el sistema únicamente en una computadora con las características adecuadas para un mejor desempeño y funcionamiento

- Mantener en constante verificación de la asistencia y permanencia de los estudiantes y constatar el abandono escolar

\section{Lista de referencias}

Castaño, M., \& Plattini, M. (1999). Fundamentos y modelos de base de datos (2 ed.). México: Alfa Omega. 
Kendall, K., \& Kendall, J. (2005). Análisis y diseño de sistemas (6 ed.). México: Pearson Educatión.

Lamarca, L. (o4 de noviembre de 2014). La interfaz gráfica. Obtenido de http://www. hipertexto.info/documentos/interfaz.htm

Laudon, K., \& Laudon, J. (2008). Sistemas de información gerencial: administración de la empresa digital (10 ed.). México: Pearson educación.

Microsoft. (13 de noviembre de 2014). Requisitos de hardware y software para instalar SQL Server 2014. Obtenido de http://msdn.microsoft.com/es-es/library/ ms143506.aspx

Peña Ayala, A. (2006). Ingeniería de Software: Una guía para crear sistemas de información (1 ed.). México D.F: Revillagigedo.

Ralph, M., \& George, W. (2013). Desarrollo de sistemas, principios de sistemas de información (1 ed.). México: Cengage Learning.

Osorio, Rivera, Fray, L. (2008). Bases de datos relacionales teoría y práctica (1 ed.). Colombia: Fondo editorial ITM.

Silberschartz, A. (2002). Fundamentos de base de datos (4 ed.). España: McGraw-Hill.

Visual Studio. (13 de noviembre de 2014). Requisitos de hardware y software para instalar visual studio 2013. Obtenido de http://www.visualstudio.com/es-es

Weitzenfeld, A. (2005). Diseño de Sistemas, ingeniería de software orientada a objetos con UML, Java e internet (1 ed.). México: Cengage Learning. 